\title{
Management of Pulmonary Interstitial Emphysema in a Premature Infant using Nasopharyngeal High-Frequency Oscillatory Ventilation
}

Khalil I. Al Tawil ${ }^{1 *}$, Ibrahim A. Ahmed ${ }^{1}$, Hesham Tawakol ${ }^{1}$, Noura Bin Saleem ${ }^{1}$, Saif A. AISaif ${ }^{1}$ and Alaa Eldemerdash ${ }^{2}$

${ }^{1}$ Department of Pediatrics, Internal mail code 1510, King Abdulaziz Medical City, Riyadh 11426, Kingdom of Saudi Arabia

${ }^{2}$ Department of Pediatrics, Alwasl Hospital, Dubai, UAE

\begin{abstract}
Pulmonary interstitial emphysema in mechanically ventilated premature infants is a serious complication that is difficult to manage and is associated with a poor prognosis [1]. We describe a premature infant with respiratory insufficiency secondary to severe pulmonary interstitial emphysema that was managed with nasopharyngeal highfrequency oscillatory ventilation, as a non-invasive mode of ventilation.
\end{abstract}

Our case report does not clearly differentiate whether the improvement in gas exchange and eventual resolution of PIE are attributable to the use of non-invasive HFOV or to the use more appropriate low frequency $(7 \mathrm{~Hz})$ with the non-invasive approach. Further studies of NP-HFOV use in infants with PIE are needed to support our report

Conclusion: NP-HFOV may be utilized in infants with PIE associated with compromised respiratory function. NPHFOV may decrease the chance of further pulmonary complications, including pneumonia and lung atelectasis, and facilitate care of sick infants while on NP-HFOV.

Keywords: Premature infants; Nasopharyngeal high-frequency oscillatory ventilation; Pulmonary interstitial emphysema

\section{Introduction}

Invasive mechanical ventilation is a cornerstone of modern neonatal units. It has contributed to the increased survival of premature infants suffering from respiratory insufficiency. However, complications such as pulmonary interstitial emphysema (PIE) occur in $3-26 \%$ of ventilated premature infants. PIE incidence correlates with delayed surfactant therapy and a lower gestational age at birth [2]. Development of PIE in mechanically ventilated very low birth weight infants (VLBW) infants is associated with an increased morbidity and mortality $[1,3]$.

High-frequency oscillatory ventilation (HFOV) delivers small tidal volumes at a supraphysiological frequency. This technique is effective in eliminating carbon dioxide and is independent of the dead space in endotracheally intubated infants.

We propose that nasopharyngeal HFOV (NP-HFOV) through a single nasopharyngeal tube can achieve adequate gas exchange, expedite extubation and the utilization of non-invasive ventilation. We describe a critically ill infant with severe PIE that was successfully managed by NP-HFOV.

\section{Case Report}

A male infant delivered via emergency Cesarean section due to maternal chorioamnionitis and fetal distress weighed $1.23 \mathrm{~kg}$ at birth and was born at 28 weeks' gestation to a 38 -year-old gravida 5, para 4 mothers.

Shortly after birth, clinical and radiological features of severe respiratory distress syndrome manifested. The patient was intubated and given 2 doses of surfactant (Survanta). Extubation was not possible because of persistence of hypoxemic respiratory failure; thus, the patient was supported using the Draeger-Babylog 8000 plus conventional ventilation in assist-control mode (Dräger Medical AG \& Co. KGaA 23542 Lübeck Germany).

During the first 12 hours of life, the patient's cardio-respiratory status was unstable, and $\mathrm{FiO}_{2}$ needs exceeded $80 \%$ with sub-adequate saturation and difference greater than $20 \%$ across the ductus arteriosus.
Dopamine, dobutamine, vasopressin, and hydrocortisone were given at maximum therapeutic doses; systemic antibiotics (Meropenim and Ampicillin) were also administered. At 8 hours of age, nitric oxide and the Sensor Medics 3100A HFOV (SM-HFOV) were started (Sensor Medics Corporation, 22705 Savi Ranch Parkway, Yorba Linda, California 92887). The inotropes and nitric oxide were gradually withdrawn and discontinued by 7 days of age.

Through the subsequent 2 weeks, chest $\mathrm{x}$-rays showed a persistence of bilateral PIE and wandering lung atelectasis (Figure 1); however, the patient maintained acceptable blood gases. Additionally, the patient failed 2 trials of extubation to nasal intermittent mandatory ventilation (N-IMV) and did not tolerate conventional ventilation. At 12 days of age; an empirical 48-hour course of dexamethasone was given to alleviate possible laryngeal edema but the bay failed extubation. The lowest SM-HFOV support settings needed during the first 2 weeks of life were as follows: $\mathrm{FiO}_{2} 2.55 \%$, MAP $7 \mathrm{~cm}$, frequency $13 \mathrm{~Hz}$, Delta $\mathrm{P}$ $24 \mathrm{~cm}$.

At the age of 19 days, the high-frequency mode of the Draeger Babylog 8000 plus ventilator was tried, but $75 \% \mathrm{FiO}_{2}$ was required. The patient accumulated a high PCO2 $(76 \mathrm{mmHg})$ due to pulmonary interstitial emphysema, lung hyperinflation and right middle and lower lobe atelectasis, as seen on the chest X-ray (Figure 1). After a thorough discussion within the treatment team, he was extubated to nasopharyngeal SM-HFOV (NP-SM-HFOV). A 3-French endotracheal tube was inserted through the nostril to a depth of $6 \mathrm{~cm}$, allowing the

${ }^{*}$ Corresponding author: Dr. Khalil AITawil, MD, Pediatric Department, Interna mail code 1510, King Abdulaziz Medical City, Riyadh 11426, Kingdom of Saudi Arabia, Tel: 00966503107188 ; Fax: 0096612520088 Extension 11641; E-mail: Tawilkio@yahoo.com

Received September 05, 2011; Accepted November 29, 2011; Published December 01, 2011

Citation: Al Tawil KI, Ahmed IA, Tawakol H, Saleem NB, AISaif SA, et al. (2011) Management of Pulmonary Interstitial Emphysema in a Premature Infant using Nasopharyngeal High-Frequency Oscillatory Ventilation. J Pulmonar Respirat Med 1:108. doi:10.4172/2161-105X.1000108

Copyright: (C) $2011 \mathrm{Al}$ Tawil KI, et al. This is an open-access article distributed under the terms of the Creative Commons Attribution License, which permits unrestricted use, distribution, and reproduction in any medium, provided the original author and source are credited. 
Citation: Al Tawil KI, Ahmed IA, Tawakol H, Saleem NB, AISaif SA, et al. (2011) Management of Pulmonary Interstitial Emphysema in a Premature Infant using Nasopharyngeal High-Frequency Oscillatory Ventilation. J Pulmonar Respirat Med 1:108. doi:10.4172/2161-105X.1000108

tip of the tube to show in the lower pharynx. The initial setup of the ventilator was as follows: MAP of $10 \mathrm{~cm}$, Delta P $28 \mathrm{~cm}$, Frequency 7 $\mathrm{Hz}, \mathrm{FiO} 2$ 75\%. The patient had good chest movement, and the chest $\mathrm{X}$-ray showed good lung inflation. Extubation was well tolerated with satisfactory oxygen saturation and acceptable blood gases. NP-SMHFOV was kept for 11 days, and the patient had acceptable PCO2 values (Figure 2) decreasing oxygen requirements (Figure 3) and disappearance of PIE changes (Figure 4). Once the oxygen requirement and NP-SM-HFOV support were low, the patient was shifted to conventional N-IMV, which was well tolerated.

The N-IMV was discontinued 8 days later. Other relevant investigations included the following: the blood culture taken at birth yielded Streptococcus viridans within 36 hours; cranial ultrasound at 5 days of age showed a bilateral grade 3 intraventricular hemorrhage that was static; and the diagnosis and subsequent management of bronchopulmonary dysplasia.

The patient was discharged home in good health at 97 days of age. Outpatient follow-up at 5 months was significant for wheezing managed at outpatient clinics, prominent lateral ventricles on cranial ultrasound, and a normal hearing evaluation and eye exam. At 30 months of age, the patient appeared to be a healthy, growing child with a normal development for his age.

\section{Discussion}

Although invasive mechanical ventilation is frequently used to treat respiratory insufficiency or apnea in very low birth weight infants (VLBW, birth weight less than $1500 \mathrm{~g}$ ), it can be associated with serious complications including pulmonary interstitial emphysema (PIE). Even during minimal pressure ventilation [2], PIE may complicate

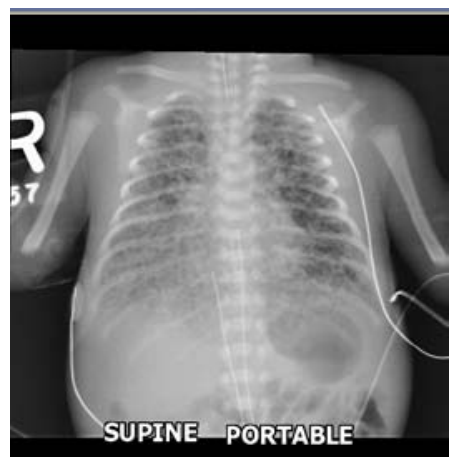

Figure 1: Chest $x$-ray of the infant at 2 days of age, showing bilateral severe PIE and atelectasis of the right middle and lower lung lobes.

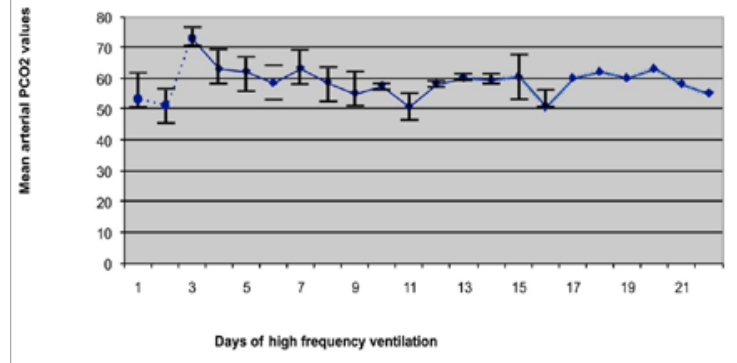

$\because:=$ Naseal HFO

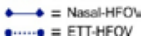

Figure 2: The relation of mean arterial $\mathrm{PCO} 2$ values and days of nasal highfrequency ventilation.

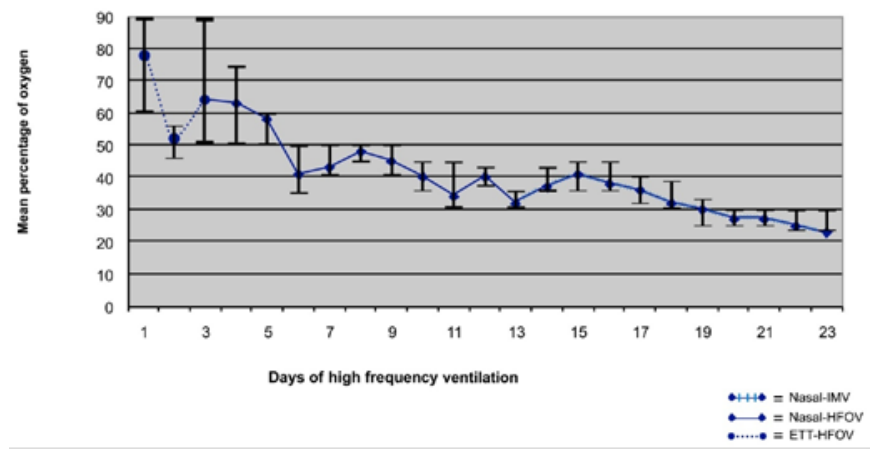

Figure 3: The relation of mean daily needed oxygen therapy to the days of nasal high-frequency ventilation.

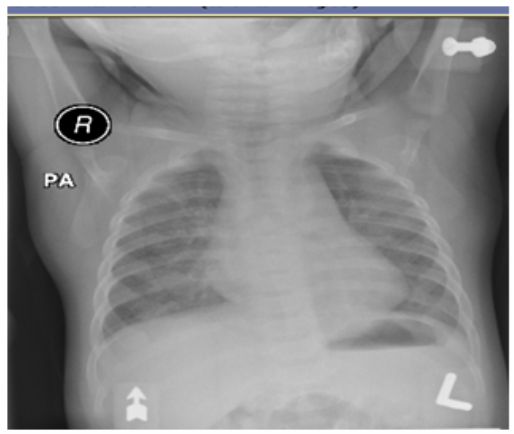

Figure 4: Chest x-ray of the infant 10 days after NP-SM-HFOV, showing the disappearance of PIE

invasive mechanical ventilation very early in the life of VLBW infants [4] with underdeveloped lungs.

Once PIE develops, the overall lung volume is increased; however, the lungs are less compliant because they are splinted at a large volume by the air within the interstitium. The increased distance between the pulmonary vascular bed and airspaces reduces gas exchange. The heart tends to get smaller as intrathoracic pressure increases, resulting in a diminished venous return and cardiac output [4].

Treatment options for PIE are limited. The only medications reported to have a role in the management of PIE are steroids [5] Minimal pressure conventional ventilation and high-frequency ventilation are the mainstays of PIE management, but positioning and ECMO may also have a role. Other treatments, such as selective bronchial intubation or surgical pleurectomy, have been tried in the past with little success [6,7].

Non-invasive respiratory support, such as nasal CPAP (N-CPAP), was reported to help preterm infants suffering from mild hypoxia associated with PIE [8].

High-frequency ventilation (HFV) is superior to conventional mechanical ventilation in managing newborns with pulmonary interstitial emphysema [9]. HFV decreases interstitial air leak as it provides adequate ventilation at lower peak distal airway pressures [10].

It has been shown in a testing trial that nasal high-frequency percussive ventilation is superior to N-CPAP in the management of TTN in term and near-term infants [11]. The efficacy of nasal HFV in decreasing $\mathrm{PCO}_{2}$ in premature infants with mild respiratory 
Citation: Al Tawil KI, Ahmed IA, Tawakol H, Saleem NB, AISaif SA, et al. (2011) Management of Pulmonary Interstitial Emphysema in a Premature Infant using Nasopharyngeal High-Frequency Oscillatory Ventilation. J Pulmonar Respirat Med 1:108. doi:10.4172/2161-105X.1000108

distress has already been reported $[12,13]$. Our case report is unique in demonstrating the effectiveness of the Sensor Medics NP-HFOV in the gas exchange of unstable, seriously ill infants with PIE and no additional risks of increased morbidity or mortality.

\section{Conclusion}

NP-HFOV may be utilized in infants with PIE associated with compromised respiratory function. Further studies of NP-HFV use in infants with PIE are needed to support our report. NP-HFV may decrease the chance of further pulmonary complications, including pneumonia and lung atelectasis, and facilitate care of sick infants while on NP-HFOV.

\section{Acknowledgement}

The authors would like to thanks King Abdullah International Medical Research Center (KAIMRC), King Saud bin Abdulaziz University for Health Sciences for the editorial assistance.

\section{References}

1. Price JF, Hart SM, McNair M (1983) Pulmonary interstitial emphysema in very low birth weight infants. Arch Dis Child 58: 612-615.

2. Bhatt JA, Ryan MR (2011) Pulmonary Interstitial Emphysema. Medscape Reference.

3. Cochran DP, Pilling DW, Shaw NJ (1994) The relationship of pulmonary interstitial emphysema to subsequent type of chronic lung disease. $\mathrm{Br} \mathrm{J}$ Radio 67: 1155-1157
4. Morisot C, Kacet N, Bouchez MC, Rouland V, Dubos JP, et al. (1990) Risk factors for fatal pulmonary interstitial emphysema in neonates. Eur J Pediatr 149: 493-495.

5. Mohsini K, Reid D, Tanswell K (1987) Resolution of acquired lobar emphysema with dexamethasone therapy. J Pediatr 111: 901-904.

6. Gaylord MS, Thieme RE, Woodall DL, Quissell BJ (1985) Predicting mortality in low-birth-weight infants with pulmonary interstitial emphysema. Pediatrics 76: $219-224$.

7. Campbell AN, Zarfin Y, Perlman M (1984) Selective bronchial intubation for pulmonary emphysema. Arch Dis Child 59: 890-892.

8. Al-Abdi YS, Singhal N (2005) Pulmonary interstitial emphysema and continuous positive airway pressure in a premature infant. Saudi Med J 26: 1627-1629.

9. Keszler M, Donn SM, Bucciarelli RL, Alverson DC, Hart M, et al. (1991) Multicenter controlled trial comparing high-frequency jet ventilation and conventional mechanical ventilation in newborn infants with pulmonary interstitial emphysema. J Pediatr 119: 85-93.

10. Clark RH, Gerstmann DR, Null DM, Yoder BA, Cornish JD, et al. (1986) Pulmonary interstitial emphysema treated by high-frequency oscillatory ventilation. Crit Care Med 14: 926-930.

11. De La Roque DE, Bertrand C, Tandonnet O, Rebola M, Roquand E, et al. (2011) Nasal high frequency percussive ventilation versus nasal continuous positive airway pressure in transient tachypnea of the newborn: A pilot randomized controlled trial. Pediatr Pulmonol 46: 218-223.

12. Colaizy TT, Younis MU, Bell FE, Klein MJ (2008) Nasal high-frequency ventilation for premature infants. Acta Paediatr 97: 1518-1522.

13. Mark van der Hoeven, Erik Brouwer, Carlos E Blanco (1998) Nasal high frequency ventilation in neonates with moderate respiratory insufficiency. Arch Dis Child Fetal Neonatal Ed 79: F61-F63. 